\title{
NUMERICAL INVESTIGATION OF DYNAMIC STALL OF AN OSCILLATING AEROFOIL
}

\author{
W. H. GUO, D. X. FU AND Y. W. MA \\ Institute of Mechanics, Chinese Academy of Sciences, Beijing 100080, China
}

\begin{abstract}
SUMMARY
The flow structure around an NACA 0012 aerofoil oscillating in pitch around the quarter-chord is numerically investigated by solving the two-dimensional compressible $\mathrm{N}-\mathrm{S}$ equations using a special matrix-splitting scheme. This scheme is of second-order accuracy in time and space and is computationally more efficient than the conventional flux-splitting scheme. A 'rigid' C-grid with $149 \times 51$ points is used for the computation of unsteady flow. The freestream Mach number varies from 0.2 to 0.6 and the Reynolds number from 5000 to 20,000 . The reduced frequency equals $0.25-0.5$. The basic flow structure of dynamic stall is described and the Reynolds number effect on dynamic stall is briefly discussed. The influence of the compressibility on dynamic stall is analysed in detail. Numerical results show that there is a significant influence of the compressibility on the formation and convection of the dynamic stall vortex. There is a certain influence of the Reynolds number on the flow structure. The average convection velocity of the dynamic stall vortex is approximately 0.348 times the freestream velocity.
\end{abstract}

KEY wORDS Dynamic stall Unsteady flow Vortex Compressible flow Matrix-splitting scheme N-S equations

\section{INTRODUCTION}

The problem of dynamic stall has been a topic of great interest to aerodynamicists and scientists. First, this problem presents a unique combination of unsteady effects, flow non-linearity and strong viscous-inviscid interaction, so it is an important theoretical problem. Second, it is of practical importance in various aerodynamic applications, including aircraft manoeuvrability, helicopter rotors and wind turbines. The challenging and difficult features of dynamic stall have stimulated its study in numerical, analytical and experimental areas.

In Reference 1 some basic features of the viscous-inviscid interaction in dynamic stall were described and these serve as a guide for numerical simulations. Reference 2 presented an important review of the analysis and prediction of dynamic stall. In recent years, with the development of high-quality computational methods, the direct numerical simulation of dynamic stall by solving the $\mathrm{N}-\mathrm{S}$ equations has become an efficient method and several works have been published. ${ }^{3-6}$ The incompressible cases of dynamic stall have been studied in detail in the past years, but compressibility effects on dynamic stall are still an unclear problem. ${ }^{2}$ In addition, little is known of the influence of the Reynolds number on dynamic stall. Thus there is still much to be learned about dynamic stall. Mach number effects on the dynamic stall vortex have been studied experimentally by Chandrasekhara and Carr. ${ }^{7}$ Usually it is difficult to determine the influences of the Mach number and the Reynolds number on dynamic stall separately by experiment, but it is easy to do so by numerical simulation. Because of these reasons, the flow structure of an oscillating NACA 0012 aerofoil is numerically studied by solving the $\mathrm{N}-\mathrm{S}$ equations in this paper. The compressibility influence on dynamic stall is studied in detail and 
the influence of the Reynolds number of dynamic stall is briefly discussed. In the following sections we first outline the numerical procedure, then the computational results are analysed.

\section{NUMERICAL PROCEDURE}

The two-dimensional, unsteady compressible $\mathrm{N}-\mathrm{S}$ equations can be written in a conservation form in a curvilinear co-ordinate system as ${ }^{8}$

$$
\partial_{\tau} q+\partial_{\xi} E+\partial_{\eta} F=\operatorname{Re}^{-1}\left(\partial_{\xi} R+\partial_{\eta} S\right)
$$

To solve equation (1), the second-order-accurate time difference is used. The difference equations are written in the implicit form

$$
\left(I+\frac{\Delta \tau}{2} \partial_{\xi} A+\frac{\Delta \tau}{2} \partial_{\eta} B\right) \Delta q=\Delta \tau\left[-\partial_{\xi} E-\partial_{\eta} F+R e^{-1}\left(\partial_{\xi} R+\partial_{\eta} S\right)\right],
$$

where

$$
A=\partial E / \partial q, \quad B=\partial F / \partial q
$$

On the right-hand side of equation (2) the flux terms and viscous terms are all central-differenced with second-order accuracy. The fourth-order dissipative terms ${ }^{8}$ are explicitly added to the right-hand side of equation (2) to inhibit numerical instability.

Because the computing time of the unsteady flow field is very long, it is necessary to develop an efficient scheme. Thus a special Jacobian-matrix-splitting scheme is proposed to solve the implicit parts of equation (2). The following is the main idea of the scheme.

The Jacobian matrices $A$ and $B$ are split into two parts $A^{+}, A^{-}$and $B^{+}, B^{-}$respectively according to the eigenvalue sign. However, unlike conventional splitting where $\lambda^{+}=(\lambda+|\lambda|) / 2$ and $\lambda^{-}=(\lambda-|\lambda|) / 2$, we only require $\lambda^{+} \geqslant 0, \lambda^{-} \leqslant 0$ and $\lambda^{+}+\lambda^{-}=\lambda$ in order to reduce the CPU time. On the other hand, the matrix-splitting scheme must satisfy stability and accuracy. To satisfy these demands, the following matrix splitting is proposed:

$$
\begin{aligned}
\lambda\left(A^{+}\right)=U / 2+\alpha a_{\xi}, & \lambda\left(B^{+}\right)=V / 2+\beta a_{\eta}, \\
A^{+}=\lambda\left(A^{+}\right) I, & A^{-}=A-A^{+}, \\
B^{+}=\lambda\left(B^{+}\right) I, & B^{-}=B-B^{+},
\end{aligned}
$$

where

$$
\begin{array}{cc}
U=\xi_{t}+\xi_{x} u+\xi_{y} v, & V=\eta_{t}+\eta_{x} u+\eta_{y} v, \\
a_{\xi}=a \sqrt{ }\left(\xi_{x}^{2}+\xi_{y}^{2}\right), & a_{\eta}=a \sqrt{ }\left(\eta_{x}^{2}+\eta_{y}^{2}\right)
\end{array}
$$

and $a$ is the speed of sound. The values of $\alpha$ and $\beta$ are determined according to the condition that eigenvalues of $A^{+}, B^{+}$are positive and those of $A^{-}, B^{-}$are negative. Then the implicit part of equation (2) is factored into two parts

$$
\begin{gathered}
\left(I+g \Delta_{\xi} A^{-}+h \Delta_{\eta} B^{-}\right) \Delta q^{*}=\text { RHS } \\
\left(I+g \nabla_{\xi} A^{+}+h \nabla_{\eta} B^{+}\right) \Delta q=\Delta q^{*}
\end{gathered}
$$


Table I

\begin{tabular}{cllll}
\hline Eigenvalue & \multicolumn{1}{c}{$A$} & \multicolumn{1}{c}{$A^{+}$} & \multicolumn{1}{c}{$A^{-}$} & \multicolumn{1}{c}{$C$} \\
\hline$\lambda_{1}$ & $U$ & $U / 2+\alpha a_{\xi}$ & $U / 2-\alpha a_{\xi}$ & $2 \alpha a_{\xi}$ \\
$\lambda_{2}$ & $U$ & $U / 2+\alpha a_{\xi}$ & $U / 2-\alpha a_{\xi}$ & $2 \alpha a_{\xi}$ \\
$\lambda_{3}$ & $U+a_{\xi}$ & $U / 2+\alpha a_{\xi}$ & $U / 2-(\alpha-1) a_{\xi}$ & $(2 \alpha-1) a_{\xi}$ \\
$\lambda_{4}$ & $U-a_{\xi}$ & $U / 2+\alpha a_{\xi}$ & $U / 2-(\alpha+1) a_{\xi}$ & $(2 \alpha+1) a_{\xi}$ \\
\hline
\end{tabular}

Table II

\begin{tabular}{cllll}
\hline Eigenvalue & \multicolumn{1}{c}{$B$} & \multicolumn{1}{c}{$\boldsymbol{B}^{+}$} & \multicolumn{1}{c}{$\boldsymbol{B}^{-}$} & \multicolumn{1}{c}{$\boldsymbol{D}$} \\
\hline$\lambda_{1}$ & $V$ & $V / 2+\beta a_{\eta}$ & $V / 2-\beta a_{\eta}$ & $2 \beta a_{\eta}$ \\
$\lambda_{2}$ & $V$ & $V / 2+\beta a_{\eta}$ & $V / 2-\beta a_{\eta}$ & $2 \beta a_{\eta}$ \\
$\lambda_{3}$ & $V+a_{\eta}$ & $V / 2+\beta a_{\eta}$ & $V / 2-(\beta-1) a_{\eta}$ & $(2 \beta-1) a_{\eta}$ \\
$\lambda_{4}$ & $V-a_{\eta}$ & $V / 2+\beta a_{\eta}$ & $V / 2-(\beta+1) a_{\eta}$ & $(2 \beta+1) a_{\eta}$ \\
\hline
\end{tabular}

$\Delta_{\xi}, \Delta_{\eta}$ and $\nabla_{\xi}, \nabla_{\eta}$ are forward and backward differences respectively, $g=\Delta \tau / 2 \Delta \xi$ and $h=\Delta \tau / 2 \Delta \eta$. Writing out equation (4), we get

$$
\begin{gathered}
\left(I-g A^{-}-h B^{-}\right)_{i, j} \Delta q_{i, j}^{*}=\text { RHS }-g\left(A^{-} \Delta q^{*}\right)_{i+1, j}-h\left(B^{-} \Delta q^{*}\right)_{i, j+1}, \\
\left(I+g A^{+}+h B^{+}\right)_{i, j} \Delta q_{i, j}=\Delta q_{i, j}^{*}+g\left(A^{+} \Delta q\right)_{i-1, j}+h\left(B^{+} \Delta q\right)_{i, j-1}
\end{gathered}
$$

Let $C=A^{+}-A^{-}$and $D=B^{+}-B^{-}$. The differences between the above schemes and the central difference scheme in the implicit parts are just second-order dissipative terms

$$
-\left(g \delta_{\xi}^{2} C+h \delta_{\eta}^{2} D\right) \Delta q
$$

If the eigenvalues of $C$ and $D$ are positive, they can inhibit numerical instability. The eigenvalues of all matrices for the special matrix-splitting scheme are given in Tables I and II. In fact, if $\alpha$ and $\beta$ are greater than $\frac{1}{2}$, the eigenvalues of $C$ and $D$ will be positive. The numerical stability will increase with increasing $\alpha$ and $\beta$. It can be seen from Tables I and II that the splitting (3) provides consistent dissipative quantities in the whole flow field. If other splitting forms are used, the eigenvalues of $C$ and $D$ may change with $U$ or $V$ and numerical experiments show that this is unfavourable to numerical stability. On the other hand, because equation (5b) can easily be solved through scalar operations and the plus-minus Jacobian matrices can also be computed easily, the overall computing time is greatly reduced. As a result, the special matrix-splitting scheme is computationally more efficient and leads to a more robust computation than the conventional flux-splitting scheme.

\section{BOUNDARY CONDITIONS}

Along the inflow portion of the far-field boundary, freestream conditions are specified. First-order extrapolation is used on the outflow boundary for all variables. Along the $\mathrm{C}$-grid cut, flow field values are found by averaging the variables extrapolated from above and below. 
On the aerofoil surface the following condition is applied:

$$
U=V=0 \text { or }\left(\begin{array}{l}
u \\
v
\end{array}\right)=J^{-1}\left(\begin{array}{cc}
\eta_{y} & -\xi_{y} \\
-\eta_{x} & \xi_{x}
\end{array}\right)\left(\begin{array}{c}
-\xi_{t} \\
-\eta_{t}
\end{array}\right) \text {. }
$$

The pressure on the aerofoil surface is obtained from the momentum equation by simplifying $\eta_{x} *(\xi$-momentum $)+\eta_{y} *(\eta$-momentum $):^{8}$

$$
\rho\left(\partial_{\tau} \eta_{\tau}+u \partial_{z} \eta_{x}+v \partial_{\tau} \eta_{y}\right)=\left(\eta_{x} \xi_{x}+\xi_{y} \eta_{y}\right) P_{\xi}+\left(\eta_{x}^{2}+\eta_{y}^{2}\right) P_{\eta}=\sqrt{ }\left(\eta_{x}^{2}+\eta_{y}^{2}\right) P_{n},
$$

where $n$ is the direction normal to the body surface.

The time-dependent co-ordinate transformation (i.e. moving grid) required in the present flow simulation is implemented using a 'rigid' grid attached to the aerofoil. The grid is a nearly orthogonal boundary-fitted C-grid generated by the hyperbolic technique with $149 \times 51$ grid points. The grid extends 10 chords away from the aerofoil and the minimum spacing in the $\eta$-direction is $0.0001 c$. This minimum normal spacing is small enough to allow for 10-20 grid points in the laminar boundary layer at the highest Reynolds number considered here. The non-dimensional time step $\left(t u_{\infty} / c\right)$ used is 0.002 .

\section{RESULTS AND DISCUSSION}

In order to check the numerical scheme, both steady and unsteady inviscid transonic flows are computed. It is seen from Figures 1 and 2 that both the steady and unsteady results agree well with other authors' results obtained using the central difference ${ }^{8}$ and TVD $^{9}$ schemes.

The computations of dynamic stall are performed for the NACA 0012 aerofoil oscillating in pitch about the quarter-chord. The angle of attack varies according to

$$
\alpha(t)=10^{\circ}[1-\cos (\omega t)] .
$$

The reduced frequency $K\left(=\omega c / 2 u_{\infty}\right)$ equals $0 \cdot 25-0 \cdot 5$, the Reynolds number varies from 5000 to 200,000 and the freestream Mach number from $0 \cdot 2$ to $0 \cdot 6$. Most results are taken from the first cycle of motion. The initial condition is steady flow at $0^{\circ}$ angle of attack.

The present computations are limited to low-Reynolds-number laminar flows, but are of value since they can illustrate important dynamic stall features and trends. This is particularly true in the higher-pitch-rate regime, where the energetic forcing motion is expected to temporarily dominate over any transition and turbulence effects. ${ }^{5}$

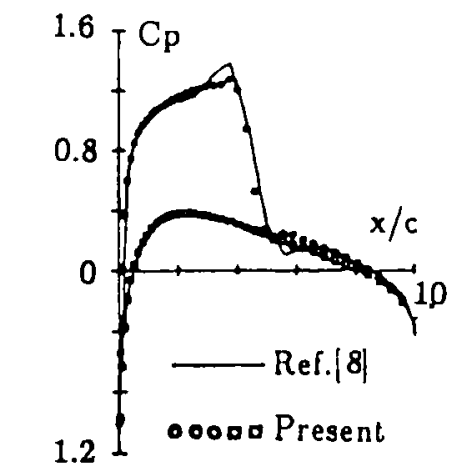

Figure 1. $C_{p}$ distribution; $M_{\infty}=0.75, \alpha=2^{\circ}$ 


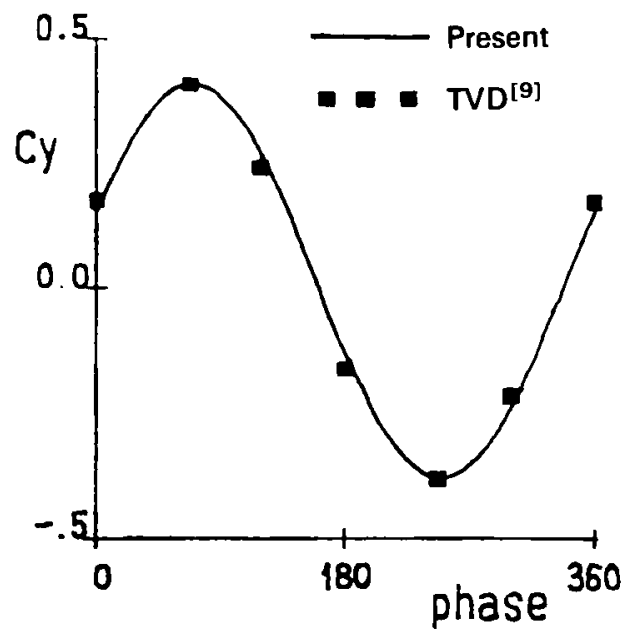

Figure 2. Unsteady lift distribution; $\alpha(t)=5^{\circ} \sin (\omega t), \omega=2, M_{\infty}=0.8$

The boundary layer separation of oscillating aerofoils can be divided into three types: ${ }^{10}$ (i) separation from the tailing edge towards the leading edge; (ii) abrupt breakdown of the turbulent flow on the forward portion of the aerofoil and followed by initial progression of flow reversal from the trailing edge to approximately the mid-chord; (iii) abrupt bursting of a leading edge laminar separation bubble. However, a dynamic stall vortex (leading edge vortex) will form at last and will dominate the features of dynamic stall no matter what type of boundary layer separation occurs. Thus the formation and convection of the dynamic stall vortex is the key phenomena to be studied here.

\subsection{Basic flow structure}

The present numerical results show that the boundary layer separation takes place from the trailing edge to the leading edge, i.e. the first type of boundary layer separation mentioned above.

Figure 3 shows the density contours in the first and second cycles of motion for the case of $R e=5000, M_{\infty}=0.4$ and $K=0.5$. The flow structure varies considerably from the upstroke to the downstroke. In the upstroke the clockwise and counterclockwise trailing edge vortices are first formed at a lower angle of attack; the leading edge vortex appears at a higher angle of attack of approximately $17^{\circ}$. From animated pictures generated by the computer we see that the leading edge vortex grows very quickly; this may be the reason why dynamic stall often occurs abruptly. Because the leading edge vortex is strong, a second counterclockwise vortex is induced near the aerofoil surface. In laminar flows even a mildly adverse gradient can produce a noticeable recirculation region which affects the local flow field. This is in contrast with high-Reynolds-number turbulent flows. ${ }^{5}$ With the movement of the leading edge vortex downstream, another small-scale leading edge vortex is shed. The interesting observation is that these vortices do not disappear in the downstroke; they still exist above the aerofoil and grow in size even though the local angle of attack may be very low. The difference in flow structure during the upstroke and downstroke processes can be seen through a comparison of Figures 3(a) and $3(\mathrm{e})$ (where $\alpha=16^{\circ}$ ) or any other pair of figures with the same angle of attack in the upstroke and downstroke in Figure 3. Figure 3(j)-3(s) show the results in the second cycle of 


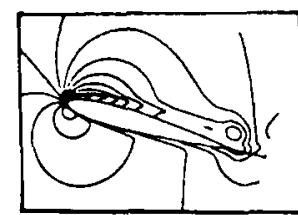

(a) up $\alpha=16^{\circ}$

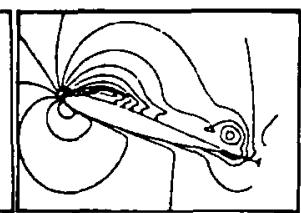

(b) up $\alpha=18^{\circ}$

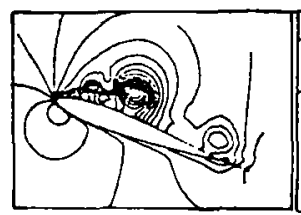

(c) up $\alpha=20^{\circ}$

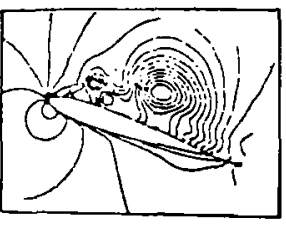

(d) down $\alpha=18^{\circ}$

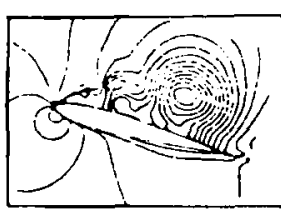

(e) down $\alpha=16^{\circ}$

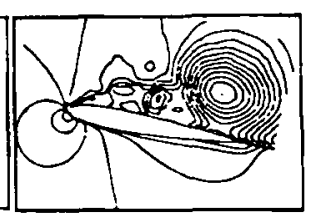

(f) down $\alpha=12^{\circ}$

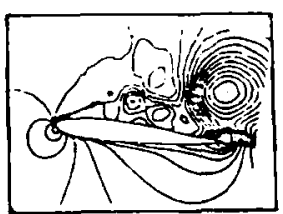

(g) down $\alpha=8^{\circ}$

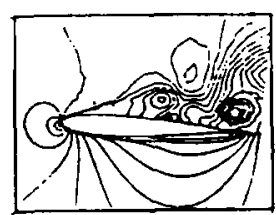

(h) down $\alpha=4^{\circ}$

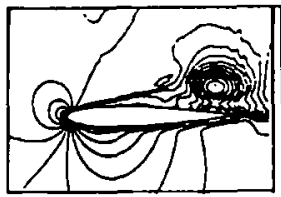

(i) down $\alpha=0^{\circ}$

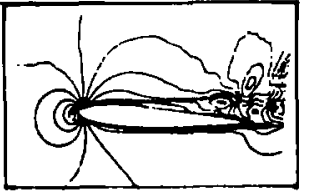

(j) up $\alpha=4^{\circ}$

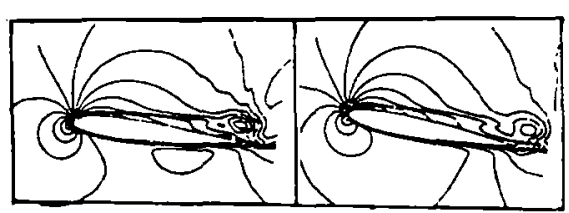

(k) up $\alpha=8^{\circ}$

(1) up $\alpha=12^{\circ}$

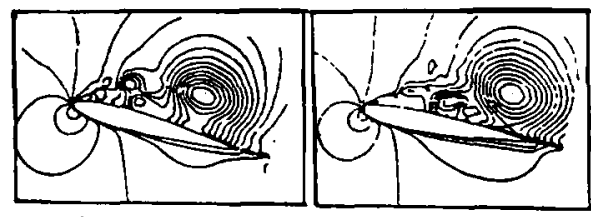

(o) down $\alpha=16^{\circ}$

(p) down $\alpha=12^{\circ}$

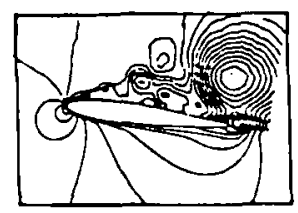

(q) down $\alpha=8^{\circ}$

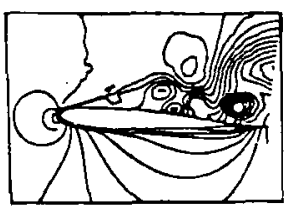

(r) down $\alpha=4^{\circ}$

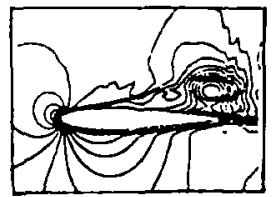

(s) down $\alpha=0^{\circ}$

Figure 3. Density contours: (a)-(i) first cycle; (j)-(s) second cycle; $M_{\infty}=0.4, \operatorname{Re}=5000, K=0.5$ 


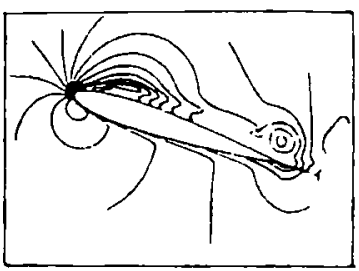

up $\alpha=18^{\circ}$

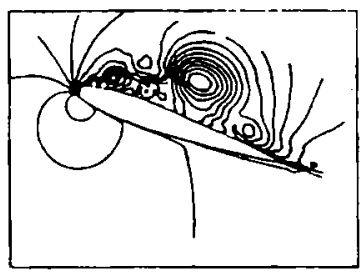

up $\alpha=20^{\circ}$

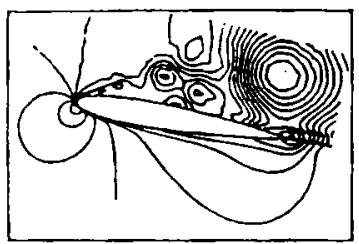

down $\alpha=10^{\circ}$

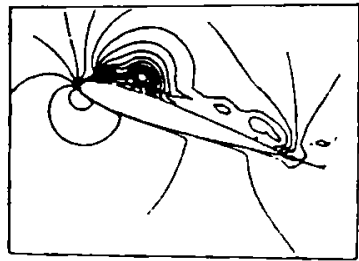

up $\alpha=18^{\circ}$

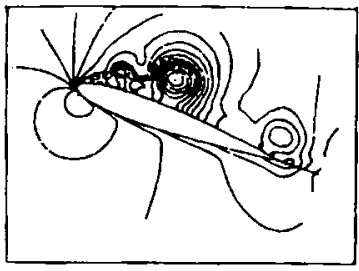

up $\alpha=20^{\circ}$

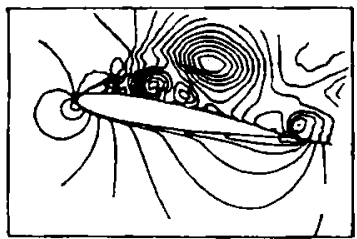

down $\alpha=10^{\circ}$

(a) $\operatorname{Re}=5000$

(b) $R_{e}=20000$

Figure 4. Density contours: $M_{\infty}=0.4, K=0.5$

motion. They are very similar to the results in the first cycle of motion and a periodic flow appears.

\subsection{Reynolds number effect}

Little has been done to determine the influence of the Reynolds number on dynamic stall, since it is difficult to vary the Reynolds number significantly without introducing a compressibility effect as well. ${ }^{2}$ However, it is possible to study the Reynolds number effect on dynamic stall by numerical simulation. Thus computations are performed for Reynolds numbers varying from 5000 to 20,000. Figure 4 shows a comparison of the results for $R e=5000$ and 20,000 . We see that the two results are different and that the leading edge vortex forms earlier for the higher-Reynolds-number case. Also, animated pictures show that the shedding rate of the leading edge vortex is higher for the higher-Reynolds-number case. Therefore the influence of the Reynolds number on the flow structure of an oscillating aerofoil cannot be neglected. Certainly, to understand the overall effects of the Reynolds number on dynamic stall, more computations 


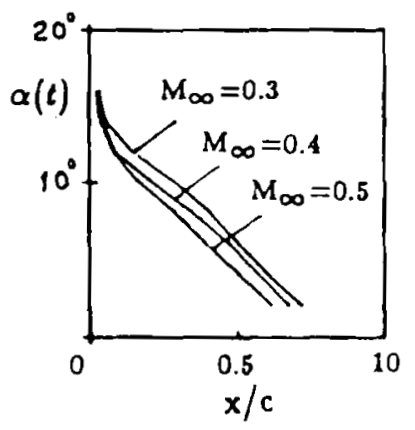

Figure 5. Location of flow reversal on surface of aerofoil; $R e=10,000, K=0.25$

are needed. Here we only provide a preliminary understanding about the effects of the Reynolds number on the flow structure.

\subsection{Compressibility effect}

To study the influence of the compressibility on dynamic stall, computations are performed for five freestream Mach numbers and two reduced frequencies. These computations are divided into two groups: the parameters of the first group are $K=0.25, R e=10,000, M_{\infty}=0.3,0.4$, 0.5 and of the second group $K=0.5, R e=5000, M_{\infty}=0.2,0.4,0.6$. It is found from the computations that the formation of the leading edge vortex is dominated by the rate of separation of the boundary layer, since the boundary layer separation takes place from the trailing edge to the leading edge and forms a strong leading edge vortex at last.

For the first-group computations Figure 5 shows that the freestream Mach number has a significant influence on the rate of boundary layer separation. With increasing Mach number the flow reversal takes place earlier. Thus the leading edge vortex forms at an earlier time and
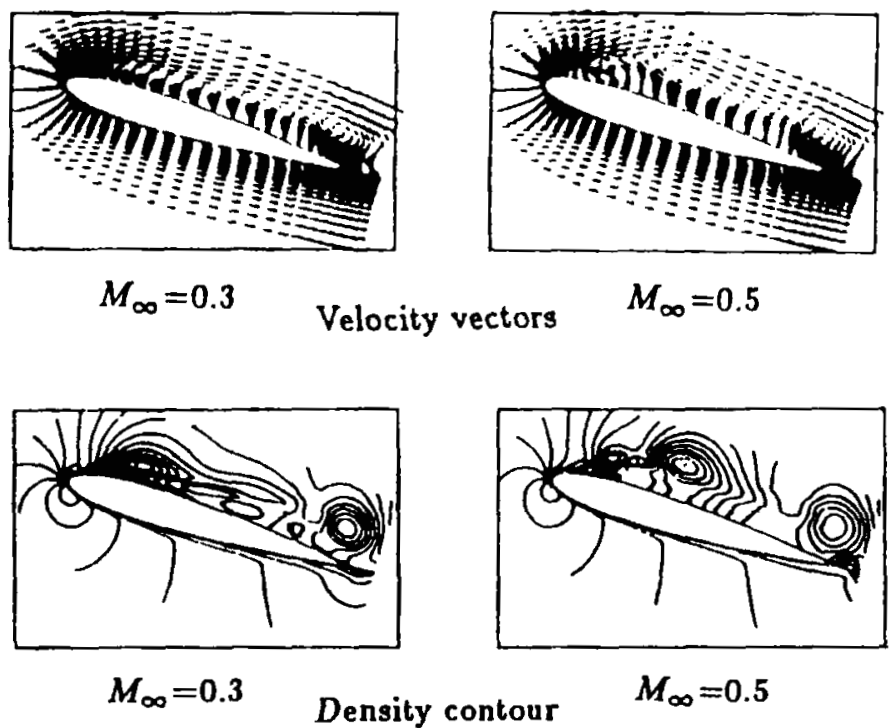

Figure 6. Velocity vectors and density contours; $R e=10,000, K=0.25, \alpha=17^{\circ}$ 


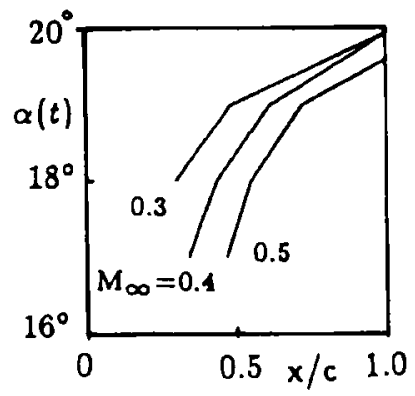

Figure 7. Location of dynamic stall vortex; $R e=10,000, K=0 \cdot 25$

this can be seen from the density contours and velocity vectors in Figure 6 . The location of the leading edge vortex is depicted in Figure 7. Increased compressibility causes the leading edge vortex to occur further downstream. Figure 8 is compiled from experimental data ${ }^{7}$ for which the variation in the angle of attack of the aerofoil is somewhat different, namely $\alpha(t)=10^{\circ}$ $[1+\sin (\omega t)]$, i.e. with a $\pi / 2$ increase in phase angle compared with here, and in addition the experimental Reynolds number is higher and the reduced frequency is lower than here. Even

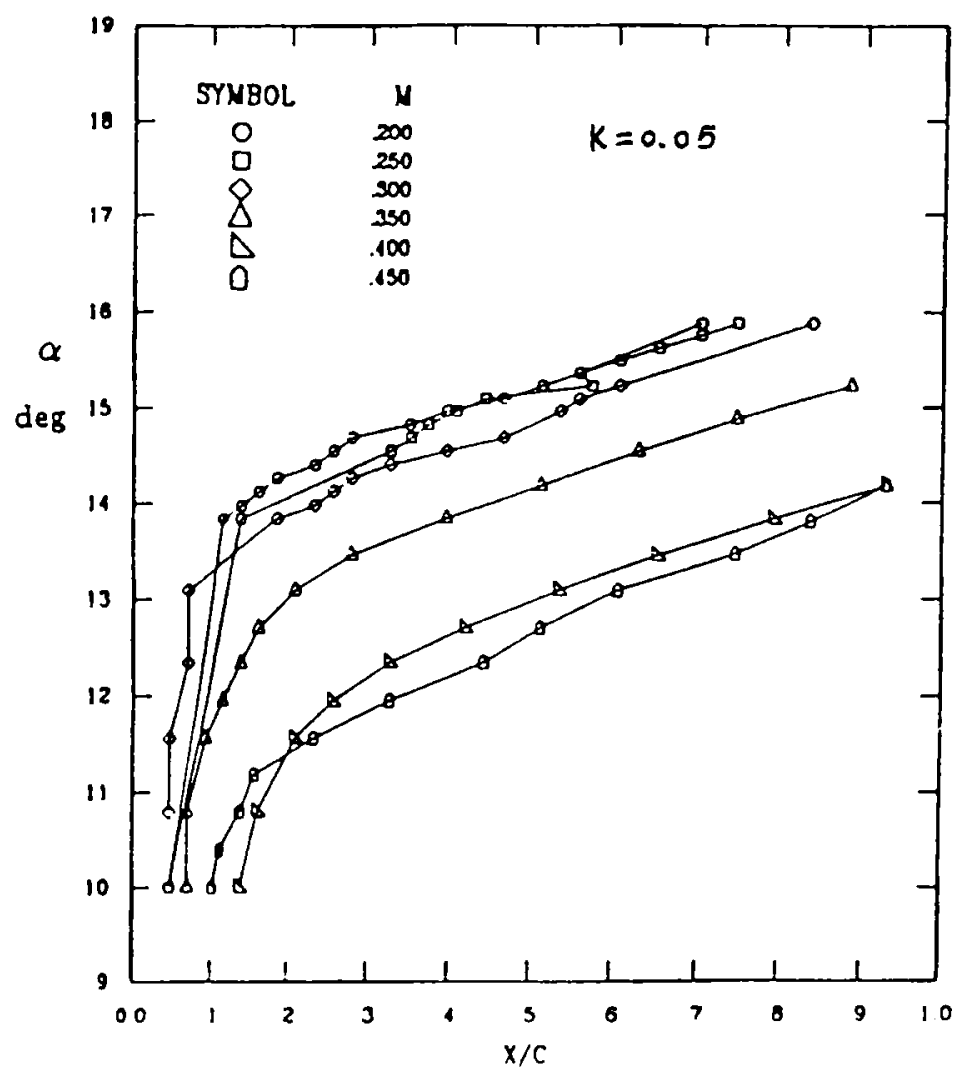

Figure 8. Location of dynamic stall vortex from Reference 7 


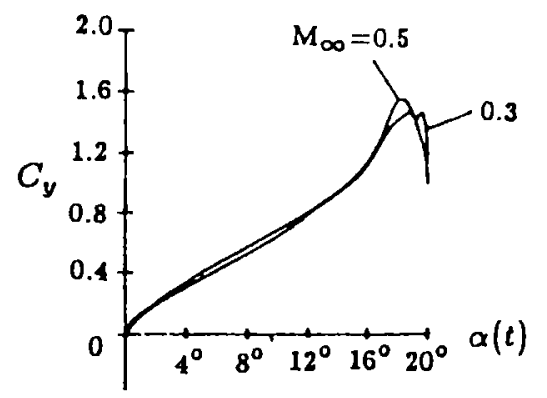

Figure 9. Lift coefficient in upstroke; $R e=10,000 ; K=0.25$

with these differences it is seen that the present numerical variation in the leading edge vortex location with the freestream Mach number is qualitatively the same as the experimental results. As a result, dynamic stall will take place earlier in the higher-Mach-number case as shown in Figure 9 , where $\alpha_{c_{\text {ymax }}}$ is smaller for the higher Mach number.

For the second-group computations, with increasing Mach number the flow reversal takes place earlier at a lower angle of attack, but the trend is opposite at a higher angle of attack (Figure 10), and this causes the leading edge vortex to form at a slightly later time (Figure 11) for the higher Mach number. In Reference 10 the same kind of result was obtained, i.e. increased compressibility has an inhibiting effect on the formation of the leading edge vortex. Therefore the rule of the influence of the compressibility on the flow structure will change with the reduced frequency. We know from previous experimental results ${ }^{2,7}$ that increased compressibility should lead to earlier leading edge vortex formation. Thus these numerical and experimental results seem to be contradictory, but now the contradiction can be explained by the present results. For most experiments the reduced frequencies are very low, so with increasing Mach number the leading edge vortex will form earlier, but for higher reduced frequencies (such as $K=0.5$ ) the result may be the opposite. It can be seen from Figure 12 that once the leading edge vortex is formed, it is also located further downstream in the higher-Mach-number case; this is the same as the results of the first-group computations.

Except as above, with increasing reduced frequency the leading edge vortex forms later. When $\alpha=20^{\circ}$, the leading edge vortex is located above the aerofoil surface for $K=0.5$ but has been shed for $K=0.25$ as shown in Figures 12 and 7.

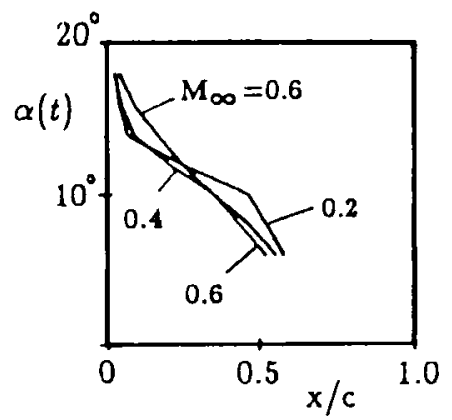

Figure 10. Location of flow reversal on surface of aerofoil; $R e=5000, K=0.5$ 


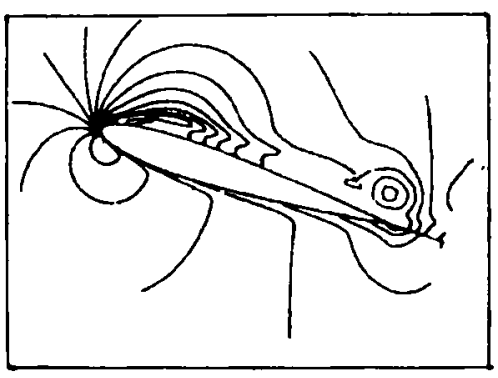

$M_{\infty}=.4$

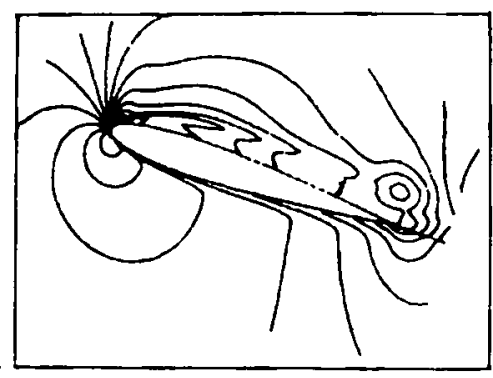

$M_{\infty}=.6$

Figure 11. Density contours; $R e=5000, K=0.5, \alpha=18^{\circ}$

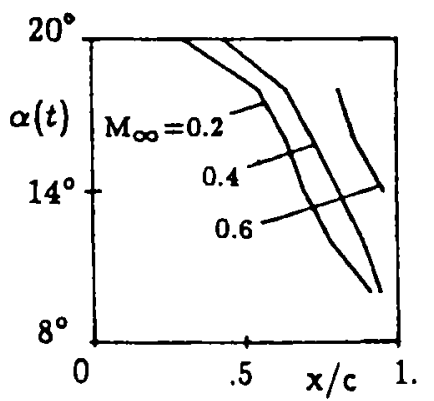

Figure 12. Location of dynamic stall vortex in downstroke; $R e=5000, K=0.5$

\subsection{Convection velocity of dynamic stall vortex}

The convection of the dynamic stall vortex can be divided into three regions: ${ }^{7}$ (i) a region where the dynamic stall vortex forms and gathers strength; (ii) a region where it convects along the surface and grows at the same time; (iii) a region where it grows and lifts off into the stream. Chandrasekhara and $\mathrm{Carr}^{7}$ obtained an average convection velocity of the dynamic stall vortex of $U_{\mathrm{DSv}} / U_{\infty}=0.3$ for the second region. From Figure 13 we see that the convection velocity of

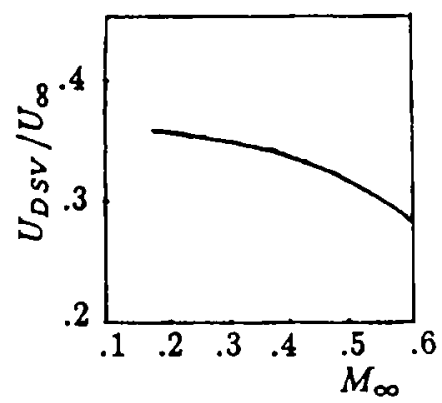

Figure 13. Convection velocity of dynamic stall vortex; $R e=5000, K=0.5$ 
Table III. Convection velocities of dynamic stall vortex

\begin{tabular}{llllll}
\hline$M_{\infty}$ & 0.2 & 0.4 & 0.6 & 0.4 & 0.5 \\
$K$ & 0.5 & 0.5 & 0.5 & 0.25 & 0.25 \\
$U_{\text {Dsv }} / U_{\infty}$ & 0.356 & 0.337 & 0.283 & 0.396 & 0.366 \\
\hline
\end{tabular}

the dynamic stall vortex decreases with increasing Mach number and that the trend is more evident when $M_{\infty}>0.4$. This variation can also be seen for the $K=0.25$ cases in Table III. The average $U_{\mathrm{Dsv}} / U_{\infty}$ of all cases studied here is $0 \cdot 348$, which is close to the experimental result of $0 \cdot 3$.

\section{CONCLUSIONS}

The dynamic stall of an oscillating NACA 0012 aerofoil is numerically investigated by solving the $\mathrm{N}$-S equations using a special matrix-splitting scheme. The scheme is of second-order accuracy in space and time with satisfied stability and is computationally more efficient than the conventional implicit matrix-splitting scheme. It is seen that the formation of the dynamic stall vortex (leading edge vortex) is related to the rate of boundary layer separation. The trailing edge vortex forms at a low angle of attack and the leading edge vortex forms at a relatively high angle of attach which is determined by the reduced frequency $K$. The influence of the Reynolds number on the flow structure cannot be neglected according to the present numerical results, which show that the leading edge vortex forms earlier and the shedding rate of the vortex is increased with increasing Reynolds number. The influence of the compressibility on dynamic stall is significant. For lower reduced frequency $(K=0.25)$ the dynamic stall vortex forms a lower angle of attack with increasing Mach number, since the boundary layer separation occurs earlier in the region of the leading edge, but the rule is opposite for higher reduced frequency $(K=0 \cdot 5)$. The convection velocity of the dynamic stall vortex decreases with increasing Mach number and the average value is approximately 0.348 times the freestream velocity.

Present research has been perfomed only for the low Reynolds number laminar flows. To understand the overall mechanisms of dynamic stall, the further research of the high Reynolds number turbulent flows is necessary. This is our next aim associated with dynamic stall research.

\section{REFERENCES}

1. W. J. McCroskey and S. I. Pucci, 'Viscous-inviscid interaction on oscillating airfoils.' AIAA Paper 81-005I, 1981.

2. L. W. Carr, 'Progress in anlaysis and prediction of dynamic stall.' J. Aircraft, 25, 6-17 (1988).

3. K. Ono, 'Numerical study on the dynamic stall process of a NACA 0012 airfoil.' AIAA Paper 85-0128, 1985.

4. Y. Shida, K. Kuwahara, K. Ono and H. Takami, 'Computation of dynamic stall of a NACA 0012 airfoil.' $A I A A$ $J$, 25, 408-413 (1987).

5. M. R. Visbal and J. S. Shang, 'Investigation of the flow structure around a rapidly pitching airfoil.' $A I A A J$., 27, 1044-1051 (1989).

6. M. R. Visbal, 'Dynamic stall of a constant-rate pitching airfoil.' J. Aircraft, 27, 400-407 (1990).

7. M. S. Chandrasekhara and L. W. Carr, 'Flow visualization studies of the Mach number effects on the dynamic stall of an oscillating airfoil.' $A I A A$ Paper $89-0023,1989$.

8. J. L. Steger, 'Implicit finite-difference simulation of flow about arbitrary two-dimensional geometries.' $A I A A J$, 16, 679-686 (1978).

9. V. Venkatakrishnan and A. Jameson, 'Computation of unsteady transonic flows by the solution of Euler equations.' AIAA J., 26, 974-981 (1988).

10. N. L. Sankar and Y. Tassa, 'Reynolds number and compressibility effects on dynamic stall of a NACA 0012 airfoil.' AIAA Paper 80-0010, 1980. 